\title{
Enterprise Level Smart Cloud Power Management System Model Based on Internet of Things and Big Data Technology
}

\author{
Zhou Xiangjun ${ }^{1, a^{*}}$, Li Hao ${ }^{2}$ \\ ${ }^{1}$ School of Information Technology, Guangdong Teachers College of Foreign Language and Arts, \\ Guangzhou, Guangdong, 510640, China \\ ${ }^{2}$ Guangzhou Think-Height Information Technology Co. Ltd, Guangzhou, Guangdong, 510640, China \\ a email: 31651989@qq.com \\ *corresponding author
}

Keywords: Internet of Things, Cloud Computing, Big Data, Energy Management

\begin{abstract}
The existing energy management platform problem description, cloud computing, big data analysis, the meaning of the Internet of things technology are analyzed, with the Internet of things industry, cloud computing platform, big data analysis, suggestions for the construction of energy management platform based on reverse optimization control. The corresponding system is developed. Practical operation shows that the system provides users with reasonable energy efficiency strategies, effectively improves energy efficiency and brings specific economic benefits.
\end{abstract}

\section{Introduction}

"12th Five Year Plan" and "13th five year plan" in the future will be an important period of lowcarbon transformation of China's industrial system [1]. At present, haze and pollutant emissions are serious, which seriously affect people's lives and our image. This situation has completely changed. In order to make "green, low-carbon development concept, energy conservation and emission reduction as the focus, establish incentive and inhibition mechanism, save resources, improve the production mode and consumption mode of environmental protection, accelerate the improvement, sustainable development" and "development capacity" are important countries in the development strategy of the 12th Five Year Plan period, and have a considerable time. As an overall outline to guide China's economic and social structure, it emphasizes the concern for the construction of a resource-based and environment-friendly society as a means of accelerating economic development [2]. The measures focus on strengthening resource protection and management, implementing key protection strategies, comprehensive resource utilization management, two-way adjustment of supply and demand and differential management. Among them, the establishment and improvement of the energy consumption online monitoring closed-loop management system of major energy consumption and emission companies is one of the important basic technical means.

\section{Internet of Things, Cloud Computing and Big Data Analysis Technology}

The progress and rapid development of cloud computing technology, data analysis and Internet technology provide a new idea to solve the problems of the above energy management system [3]. In a word, cloud computing, information technology support functions (hardware and software) are based on public facilities, and are very flexible (increase and decrease), and are provided as customer service through the network. This model realizes the high concentration of computing resources (mainly referring to three main resources: server, storage device and network). The computing resources scattered around the country are integrated into a unified resource. It is as simple as using tap water to use electricity, achieving on-demand access and payment. Intel therefore, in the era of cloud computing, this is a huge change in the manufacturing of infrastructure, computers, network equipment, or whether software changes the environment of the whole information technology industry ecology. 


\section{3. "Big data" is a Very Large Data Set}

Big data technology is a kind of technology that can quickly obtain massive data and all kinds of valuable information. Now, "big data" is not only the scale of data itself, but also the tools, platforms and data analysis systems for data collection [4]. The purpose of big data research and development is to develop big data technology, apply it to related fields, and promote its development by solving big data processing problems. In 2005, the International Telecommunication Union (ITU) released "ITU Internet Report 2005: Internet". In a broad sense, the network of things, through a variety of sensing devices and networks to connect objects and objects, anytime, anywhere, in order to achieve scientific management, information collection, and communication, as well as information processing, intelligent processing. Internet of things, IOT, interconnection, automation, perception and intelligence are the basic characteristics of Internet of things. The combination of IOT, cloud computing and big data can be used as a porting piece of new information technology of energy management system. Internet of things technology of Intel industry, cloud computing closed intelligent system technology and feedback control technology, and based on big data analysis, energy consumption equipment of process industry enterprises, online real-time energy can be supervised and managed, while data mining and energy analysis of large-scale equipment can be implemented [5]. On the basis of data collection and analysis, the actual operating conditions of the feedback energy efficiency optimization simulation model and on-site control are gathered. Through the implementation of visualization of energy consumption data, the optimization of energy consumption release is realized, and the energy conservation and emission reduction information service is provided to the enterprise energy management department and the government as the energy management organization.

Table 1 Classification of impact on enterprise value

\begin{tabular}{|c|c|c|}
\hline Classification standard & classification & concrete content \\
\hline \multirow{4}{*}{ Based on the cause of loan risk } & Credit risks & Use of bank deposit by debtor in default \\
\cline { 2 - 3 } & market risk & Economic loss caused by market price fluctuation \\
\cline { 2 - 3 } & Operational risk & Lack of bank liquidity \\
\cline { 2 - 3 } & Liquidity risk & The bank failed to fully understand the legal provisions \\
\cline { 2 - 3 } & Legal risk & Market interest rate fluctuation \\
\hline
\end{tabular}

\section{Building an Energy Management Platform Based on the Internet of Things, Cloud Computing and Big Data}

\subsection{System Architecture}

Intel products, cloud computing and large-scale data analysis based on the overall architecture of the Internet industrial enterprise energy management platform system, sensor sensing and acquisition data layer, cloud platform and big data analysis layer, and application display layer[6]. Main contents: data collection of operation parameters and energy consumption detection layer of industrial equipment, distributed control system (camera DCS) based on intersection decentralized control, big data analysis of cloud platform, relevant interfaces / protocols / standards covered. The basic idea of the system is based on the following points. Energy management platform needs to be closely related to industrial automation system. Based on the detection system, it can realize the closed-loop logic control of "detection, control, execution and feedback", combining energy management and control with energy management and control. Glue on the energy management platform software, is to meet the management, and then, the regional city industry website management needs, in order to meet the cloud computing technology, the building uses unlimited user login. From the point of view of energy conservation in back-end storage society, background investment, maintenance, management and other costs are reduced. In order to reduce, unlimited increase of storage space of guard terminal is required to meet the recruitment of cloud storage methods. To improve the collection and transmission devices of energy consumption in order to recruit technical network, each component has a unique IP address code in the world. Its cloud 
computing said convenient data is memorized by paparazzi, with processing and analysis functions [7]. It can minimize the granularity of energy consumption monitoring data, monitor the energy consumption and energy consumption trend of each energy terminal, and implement the targeted energy conservation and emission reduction measures.

Table 2 Descriptive statistical analysis

\begin{tabular}{|c|c|c|c|c|}
\hline Factor & Minimum & maximum & mean & Std.Deviation \\
\hline Product authenticity & 2.06 & 5.00 & 4.1466 & 0.49062 \\
\hline Price fairness & 132 & 4.93 & 3.2342 & 0.60204 \\
\hline High pressure promotion & 1.00 & 5.00 & 3.1356 & 0.68146 \\
\hline Technological superiority & 1.80 & 5.00 & 3.1317 & 0.886679 \\
\hline
\end{tabular}

\subsection{Data Perception Acquisition and Monitoring System}

Based on the industrial network of data sensing and monitoring system camera DCS technology, a variety of monitoring field information share the existing signals, in order to obtain the unity of control network, wireless sensor network, support and power information monitoring network. The data sensing and monitoring system is mainly divided into three sub layers: field layer, control layer and monitoring layer [8]. The field layer is mainly composed of various conventional temperature and humidity instruments, pressure sensors, flow meters, intelligent meters, actuators and intelligent electrical protection devices. The control layer is divided into three types: traditional instrument signal acquisition control, wireless sensor information acquisition and electrical monitoring information acquisition. The monitoring layer mainly includes engineer station, operator station, history station and data interface station. Data interface station is used to connect energy management cloud computing platform and data mining center. The system adopts a variety of it and industrial IOT technologies to build a complete industrial IOT solution from field data collection network to remote data transmission and open application. IOT functions of system industry include: general and special sensor acquisition system; support data transmission through fieldbus, wireless technology, etc.; please provide large capacity data storage and open application platform; please provide consistent experience based on multiple terminal devices. The integrated security strategy of information release and transaction processing, including remote collection platform, all roads of various end products, end-to-end station control of field collections, establishes a complete security system from top to bottom. Then, collect the initial industrial automation security information control of terminal controller products, and use the existing industrial merger controller and security gateway products, all kinds of external and internal real effective resistance to potential threats. At the same time, electrical control information and process control information use integrated system platform. From the perspective of the development trend of intelligent factory, at present, in the fields of power generation, chemicals, petrochemicals, steel, building materials and other industries, each industry from design institutions to factory users is divided into two specialties. The electric automatic control system is an independent policy. Through automation and process automation, the independently developed automation system operates independently, and several copied functions, the overall advantages of the automation system cannot be used. The integration of electric control system and production management system is the inevitable trend of intelligent factory in the future [9]. Control information and process control information of electrical appliances, realize information sharing, use unified system platform, better data mining, energy saving analysis, early failure alarm, and various production information of the factory, which is beneficial to the analysis of security deterioration, digitization and informatization. 


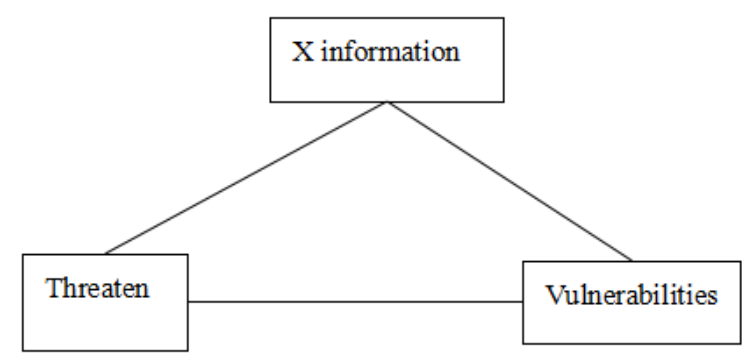

Figure 1 Enterprise smart cloud power management mode

\subsection{Data Mining Analysis and Management}

Energy consumption data analysis comprehensive energy management big data platform in these large-scale data related analysis similar to the energy consumption of websites, and finally through the discovery of change rules, the width and depth of energy consumption data is based on a wide range of collectibles. Then, the large-scale energy consumption device is a friendly data visualization demonstration method. According to the perfect cluster characteristics, this kind of energy consumption data analysis method is analyzed. Finally, the energy consumption mode of the energy consumption place is established, and the benchmark energy consumption unit is established[10]. Summarize the energy consumption law, seek energy-saving space, and provide guidance and consultation information on energy-saving trend. To achieve the purpose of energy conservation and emission reduction and help enterprises improve economic efficiency. The results of data visualization and energy data analysis are mainly displayed by flash, XML, JSON, AJAX, jQuery and other technologies. Provide general personalized data analysis services, including customized reports of drilling, winding, slicing, blocking and visual data. The cloud field and energy using area with temperature field and energy efficiency are rendered by 3D. In the aspect of rendering, it is mainly realized by 3D modeling, integrating 3D engine, and finally encapsulating and filling data with 3D model. The construction of temperature and energy efficiency cloud field is mainly based on high-density temperature and energy efficiency data. Finally, the data is generated under high-precision temperature and energy efficiency through the gradient cloud field construction algorithm based on computational fluid dynamics (CFD).

\subsection{Data Computing is Parallelized}

In the data processing part, Hadoop, an open-source distributed system architecture combined with MapReduce, and Oracle's RAC are combined to form an open system architecture. This architecture is a software framework that can process a large amount of data in a decentralized way. The distributed storage of data and the parallel computing of data analysis will provide users with efficient and accurate data analysis services. Multi test model is a data service-oriented SDO and public sustainable data engine technology, which are built to encapsulate the energy consumption of the site and the state data of the working environment. The data consolidation layer of the platform, the $7 \times 24$-hour data integrated environment of the cloud computing platform based on the data center, and the construction of social tenants or government energy management agencies to provide data services for the convenience of energy consumption. At the same time, it uses equipment monitoring information service support to provide social users of energy consumption.

\section{Conclusion}

The system combines industrial Internet, cloud computing, big data analysis and industrial DCS intelligent control. In addition, the advantages of their respective fields are given to complete games, collecting various energy consumption and release data including smart sensor sensing. Through the 
front-end DCS, the data processing of the system is uploaded online, and the final analysis of the process. Then, a large amount of energy consumption information processing is released. The linked devices of the production process use a lot of energy consumption release monitoring and energy efficiency, which provides a beneficial energy efficiency for the realization of the dynamic control strategy. The purpose of KPI data optimization control, such as large amount of energy consumption, storage and mining analysis is to provide monitoring and management of energy consumption data of real-time equipment as well as energy consumption analysis. High energy consumption industry enterprises and the government's response to the informatization and energy conservation of energy consumption management departments is an important millstone industry for the large-scale expansion of energy-saving emission monitoring and management system platform in high energy consumption.

\section{Acknowledgements}

The work described in this paper was fully supported by a grant from Characteristic innovation projects of universities in Guangdong “An enterprise-level smart cloud power management system supported by the Internet of Things and big data technologies” (Grant No.2017GKTSCX006).

\section{References}

[1] Yuhuai Peng, Xiaojie Wang, Dawei Shen,. Design and modeling of survivable network planning for software-defined data center networks in smart city. International Journal of Communication Systems, vol. 31, no. 16, 2018.

[2] Hongming Cai, Boyi Xu, Lihong Jiang,. IoT-Based Big Data Storage Systems in Cloud Computing: Perspectives and Challenges. IEEE Internet of Things Journal, vol. 4, no. 1, pp. 75-87, 2017.

[3] Mauro Femminella, Matteo Pergolesi, Gianluca Reali. IoT, big data, and cloud computing value chain: pricing issues and solutions. Annals of Telecommunications, vol. 73, no. 7-8, pp. 511-520, 2018.

[4] Bingyu Sun, Xiufang Jia, He Huang,. Decision-making and management system based on internet of things for Hulun Buir State Farm. Chinese Science Bulletin, 2018.

[5] Edward Curry, Souleiman Hasan, Christos Kouroupetroglou,. Internet of Things Enhanced User Experience for Smart Water and Energy Management. IEEE Internet Computing, vol. 22, no. 1, pp. 18-28, 2018.

[6] Farhan L, Kharel R, Kaiwartya O, et al. Towards green computing for Internet of things: Energy oriented path and message scheduling approach, vol. 38, pp. 195-204, 2018.

[7] Intae Ryoo, Kyunghee Sun, Jaesun Lee,. A 3-dimensional group management MAC scheme for mobile IoT devices in wireless sensor networks. Journal of Ambient Intelligence \& Humanized Computing, vol. 9, no. 1, pp. 1-12, 2017.

[8] Abdulhameed Alelaiwi. A collaborative resource management for big IoT data processing in Cloud. Cluster Computing, vol. 20, no. 5, 2017.

[9] Frédéric Blanchard, Hacène Fouchal, Michel Herbin. Energy and activity monitoring over wireless sensor networks. Concurrency \& Computation Practice \& Experience, vol. 29, no. 4, 2017.

[10] Marceau J. The internationalization of R\&D: contents and opportunities for developing countries, 2018. 